anatomic course in the forearm. The anomaly we have described has not been previously reported. The closest description is by Funk and colleagues $^{4}$ of a rare anomaly arising from a distal division of the brachial artery into the ulnar and RA arteries under the pronator teres muscle with the RA pedicle assuming its normal position in the distal forearm through the pronator teres muscle. In our case, the venae comitantes had a normal anatomic course above the pronator teres. The RA in its aberrant course was not accompanied by the venae comitantes.

The development of a congenital anomaly, such as a high-origin RA, has been attributed to an arrest in stage $\mathrm{V}$ of upper limb vascular development (embryos of $23 \mathrm{~mm}$ C-R length). ${ }^{5}$ The proximal venae comitantes pedicle with the band may represent a developmental regression of the proximal part of the RA. ${ }^{5}$ As reported by Funk and colleagues, ${ }^{4}$ we believed that this anomaly would not be identifiable on routine preoperative examination of the forearm. Other forearm arterial anomalies have been described that are associated with hand vascular anomalies. ${ }^{2,5}$

\section{Conclusions}

A knowledge of the major arterial variation of the upper arm should be consider essential for anyone involved in harvesting of RA conduits to help avoid unnecessary difficulties or complications.

\section{References}

1. Desai ND, Cohen EA, Naylor CD, Fremes SE. A randomized comparison of radial-artery and saphenous-vein coronary bypass grafts. $N$ Engl J Med. 2004;351:2302-9.

2. Fox AD, Whiteley MS, Phillips-Hughes J, Roake J. Acute upper limb ischemia: a complication of coronary artery bypass grafting. Ann Thorac Surg. 1999;67:535-6.

3. McCormack LJ, Cauldwell EW, Anson BJ. Brachial and antebrachial arterial patterns. A study of 750 extremities. Surg Gynecol Obstet. 1953;96:54.

4. Funk GF, Valentino J, McCulloch TM, Graham SM, Hoffman HT. Anomalies of forearm vascular anatomy encountered during elevation of the radial forearm flap. Head Neck. 1995;17:284-92.

5. Gonzalez-compta $X$. Origin of the radial artery from the axillary artery and associated hand vascular anomalies. J Hand Surg [Br]. 1991;16A:293-6.

\title{
Open stent grafting of descending aorta to control suture line hemorrhage in type B dissection
}

\author{
Imran Saeed, MRCS, Athanasios Tsiouris, MRCS, Tahir Ali, MRCS, Jean-Pierre van Besouw, FRCA, Matt Thompson, FRCS, \\ and Marjan Jahangiri, FRCS, London, United Kingdom
}

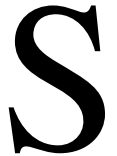
tent graft placement under direct vision has previously been described during surgical repair of type A aortic dissection to manage further entry flaps or dissection of the descending aorta. ${ }^{1,2}$ In these series, stents are deployed in an antegrade fashion with the aim of inducing thrombosis of the false lumen and pre-empting possible future complications that may arise from residual type B dissection. Kubota, Endo, and Sudo $^{3}$ have described techniques to facilitate the insertion of stents in this manner and make it safer.

We describe the use of retrograde open stent graft placement as a rescue procedure to control significant suture line hemorrhage after open repair of a descending aortic dissection.

\section{Clinical Summary}

A 29-year-old man with Marfan syndrome who had undergone aortic root replacement with a Freestyle prosthesis (Medtronic, Inc,

From the Departments of Cardiothoracic and Vascular Surgery, St George's Hospital, London, United Kingdom.

Received for publication Dec 17, 2007; revisions received Jan 23, 2008; accepted for publication Feb 2, 2008.

Address for reprints: Marjan Jahangiri, FRCS, Department of Cardiac Surgery, St George's Hospital Medical School, Blackshaw Rd, London SW17 0QT, United Kingdom (E-mail: marjan.jahangiri@stgeorges.nhs.uk).

J Thorac Cardiovasc Surg 2008;135:1390-2

$0022-5223 / \$ 34.00$

Copyright (C) 2008 by The American Association for Thoracic Surgery doi:10.1016/j.jtcvs.2008.02.039
Minneapolis, Minn) and only proximal aortic replacement 2 years previously at another hospital arrived at our emergency department with right hypochondrial pain. Computed tomographic (CT) scans showed a 9-cm infrarenal abdominal aortic aneurysm (AAA) and a 5 -cm descending thoracic aorta.

The patient underwent laparotomy, which revealed a ruptured juxtarenal AAA.

A significant pectus deformity associated with Marfan syndrome made it impossible to access the supraceliac aorta. Zenith stents (Cook Inc, Bloomington, Ind) were, therefore, deployed to cover the AAA, both iliac arteries, and the left renal artery.

After this, the patient made satisfactory progress. However, CT scans a week after the operation showed a type I endoleak and a new dissection in the descending aorta. The dissection extended from the level of the pulmonary trunk to just above the previously inserted abdominal stent graft. The diameter of the descending aorta was $5.3 \mathrm{~cm}$ and was noted to have an acute "U-bend" just above the diaphragm, rendering endovascular repair difficult. The endoleak was managed with a Palmaz stent (Johnson \& Johnson Interventional Systems, Warren, NJ) inserted via the brachial approach, and the dissection of the descending aorta was managed medically. The size of the descending aorta remained stable and the patient was discharged home.

He returned a month after discharge with back pain. CT scans at this point showed progressive expansion of the descending aorta to $6.5 \mathrm{~cm}$ associated with a moderate-sized left pleural effusion and a proximal intramural hematoma (Figure 1). Considering these new findings, we proceeded with open repair of the descending aorta. 


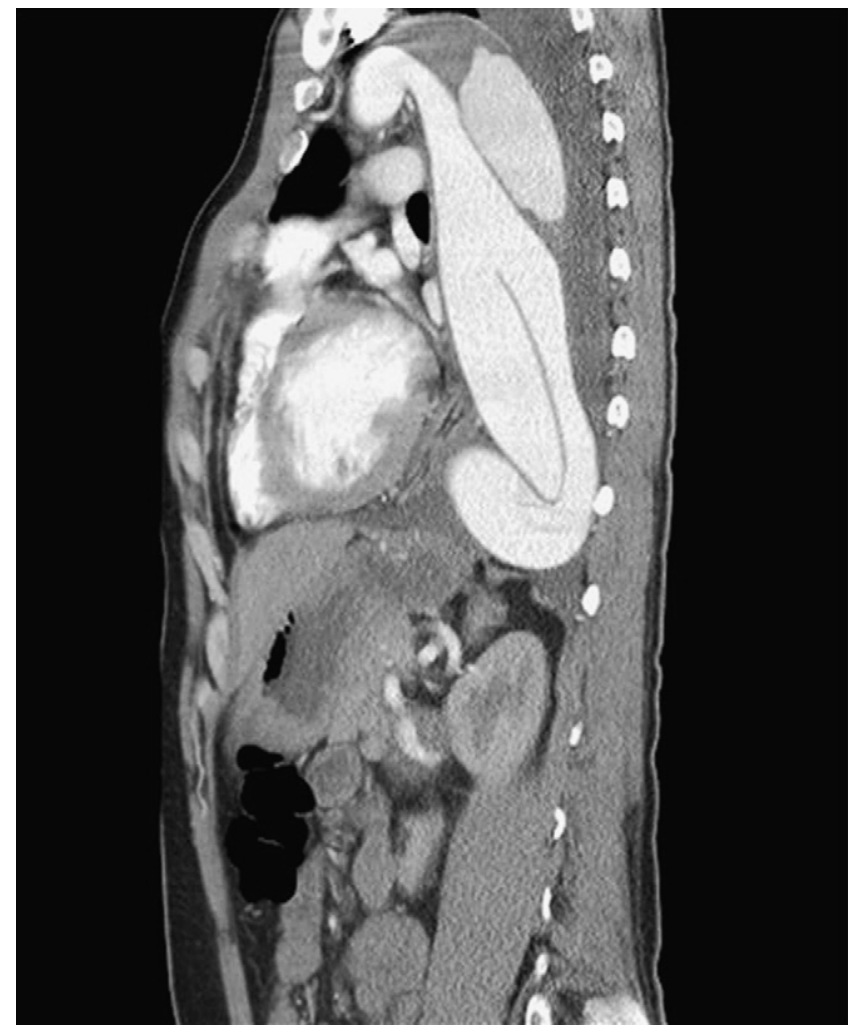

Figure 1. CT scan showing ascending aortic dissection and intramural hematoma.

Surgery was performed with cerebral spinal fluid drainage, femorofemoral bypass, cooling to $32^{\circ} \mathrm{C}$, and a left posterolateral thoracotomy. During the initial open operation, only the proximal aorta had been replaced. Therefore, consideration was not given to a graft-to-graft anastomosis. The distal arch and the proximal and distal descending aorta were mobilized, and the proximal and distal descending aorta was clamped. The descending aorta was excised and replaced with a 30-mm Dacron graft with Teflon strips to buttress the suture lines. Aortic tissue was particularly fragile in the proximal dissected descending aorta and was associated with persistent and significant bleeding around the suture line. Despite prolonged attempts at obtaining hemostasis, this could not be managed satisfactorily.

At this point, we believed that repeating the proximal anastomosis through such fragile tissue would not guarantee a secure suture line. We therefore opted to reinforce our existing suture line with an endovascular stent placed under direct vision.

A purse string was made in the Dacron graft (neoaorta). The descending neoaorta was partially occluded with a side-clamp and a 40 $\times 15$-mm Tag Stent (W. L. Gore \& Associates, Inc, Flagstaff, Ariz) was inserted via an incision in the purse string. The stent was guided to cross the proximal suture line (distal to the left subclavian artery) using a combination of direct vision, manual palpation, and transesophageal echocardiographic guidance, and then deployed. There was an almost immediate improvement in bleeding, allowing satisfactory hemostasis and chest closure.

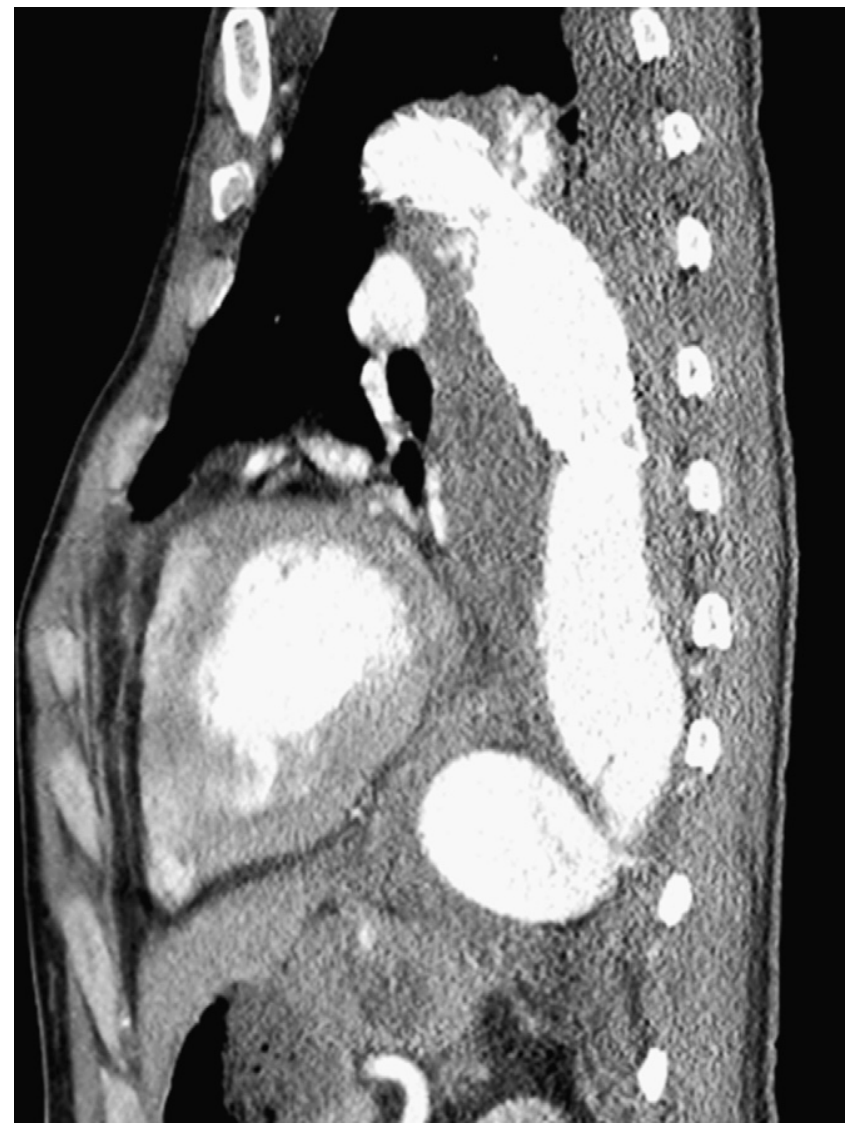

Figure 2. CT scan after surgery showing Dacron graft replacement with the stent within the graft.

The patient made a satisfactory recovery in the early postoperative period. He had signs of a brain stem stroke a week after the operation, which completely resolved spontaneously, and was discharged home 2 weeks later. A CT scan before discharge showed a well-positioned stent (Figure 2). Subsequent scans have excluded the possibility of an endoleak. The patient will be monitored, particularly for the distal part of the aorta.

\section{Discussion}

During repair of type A dissection, stenting of the descending aorta has been advocated to avoid possible long-term sequelae of residual dissection, ${ }^{1,2}$ although the absolute need and benefit of managing the descending aorta in this situation is unclear. ${ }^{4}$ We have applied this technology in a different but not uncommon surgical situation, namely, that of a difficult bleeding point in the context of poor surgical tissues. This technique is not a substitute for precise surgical suturing, but we found it to be helpful in a situation with uncontrollable bleeding in the presence of fragile tissues.

This case also highlights the importance of eliminating as much aortic tissue as possible at the time of original treatment in patients with Marfan syndrome to avoid future multiple and high-risk interventions. 


\section{References}

1. Uchida N, Isihara H, Shibamura H, Kyo Y, Ozawa M. Midterm results of extensive primary repair of the thoracic aorta by means of total arch replacement for an acute type A aortic dissection. J Thorac Cardiovasc Surg. 2006;131:862-7.

2. Kato M, Kuratani T, Kaneko M, Kyo S, Ohnishi K. The results of total arch graft implantation with open-stent graft placement for type A aortic dissection. J Thorac Cardiovasc Surg. 2002;123:1035-40.
3. Kubota H, Endo H, Sudo K. New open-stent-graft delivery system: the CLATE flexible metal graft holder. Interactive Cardiovasc Thorac Surg. 2006;5:333-5.

4. Dobrilovic N, Elefteriades JA. Stenting the descending aorta during repair of type A dissection: technology looking for an application? J Thorac Cardiovasc Surg. 2006;131:777-8.

\title{
A meta-analysis of comparative studies of endovascular versus open repair for blunt thoracic aortic injury
}

\author{
Hisato Takagi, MD, PhD, Norikazu Kawai, MD, and Takuya Umemoto, MD, PhD, Shizuoka, Japan
}

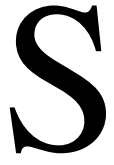

everal recent comparative studies $^{1-5}$ of endovascular (EVR) versus open repair (OR) for blunt thoracic aortic injury (BTAI) suggest that EVR may be associated with a reduction in mortality. Such comparisons are, however, hampered by the small number of cases, owing to the relative rarity of this condition. ${ }^{4}$ Therefore, the appropriate role of EVR for BTAI remains unclear. We performed a meta-analysis of all comparative studies of EVR versus OR for BTAI to date.

\section{Materials and Methods}

All comparative studies of EVR versus OR for BTAI were identified by a 2-level search strategy. First, a public domain database (MEDLINE) was searched using a Web-based search engine (PubMed). Second, relevant studies were identified through a manual search of secondary sources including references of initially identified articles and a search of reviews and commentaries. The MEDLINE database was searched from January 1966 to December 2007. MeSH keywords included "Aortic Rupture," "Aorta," "Wounds and Injuries," "Aortic Aneurysm, Thoracic," "Stents," and "Comparative Study." Studies considered for inclusion met the following criteria: the design was a comparative study of EVR versus OR; the study population was patients with BTAI; and main outcomes included mortality. Data regarding detailed inclusion criteria and mortality were abstracted (as available) from each individual study. For each study, data regarding mortality in both the EVR and OR groups

From the Department of Cardiovascular Surgery, Shizuoka Medical Center, Shizuoka, Japan.

Received for publication Dec 19, 2007; accepted for publication Jan 13, 2008.

Address for reprints: Hisato Takagi, MD, PhD, Department of Cardiovascular Surgery, Shizuoka Medical Center, 762-1 Nagasawa, Shimizu-cho, Suntogun, Shizuoka 411-8611, Japan (E-mail: kfgth973@ybb.ne.jp).

J Thorac Cardiovasc Surg 2008;135:1392-4

$0022-5223 / \$ 34.00$

Copyright $\odot 2008$ by The American Association for Thoracic Surgery doi:10.1016/j.jtcvs.2008.01.033 were used to generate odds ratios and $95 \%$ confidence intervals (CIs). Study-specific estimates were combined using inverse variance-weighted averages of logarithmic odds ratios in a random-effects model. Between-study heterogeneity was analyzed by standard $\chi^{2}$ tests. Sensitivity analyses were performed to assess the contribution of each study to the pooled estimate by excluding individual studies one at a time and recalculating the pooled odds ratio estimates for the remaining studies. Publication bias was assessed graphically by a funnel plot and mathematically by an adjusted rank-correlation test.

\section{Results}

Our search identified 17 retrospective nonrandomized comparative studies $^{1-5, \text { E1-E12 }}$ of EVR versus OR for BTAI. The baseline patient characteristics are summarized in Table 1. In $11^{1-5, \mathrm{E} 3, \mathrm{E} 4, \mathrm{E} 7, \mathrm{E} 8, \mathrm{E} 11, \mathrm{E} 12}$ of the 17 studies, the EVR and OR groups had similar preoperative variables including the injury severity score. Fifteen of the 17 individual studies demonstrated a statistically nonsignificant benefit of EVR over OR for mortality, whereas only one study ${ }^{\mathrm{E} 10}$ demonstrated a statistically nonsignificant mortality reduction with OR over EVR. Pooled analysis of all the 17 studies (representing 565 patients) demonstrated a statistically significant $57 \%$ reduction in mortality with EVR relative to OR $(8.1 \%$ in the EVR group vs $20.8 \%$ in the OR group; odds ratio, 0.43 ; $95 \%$ CI, $0.25-0.76 ; P<.01$ ) (Figure 1, upper panel). There was neither study heterogeneity of results $(P=.96)$ nor evidence of significant publication bias $(P=.32)$. To assess the impact of qualitative heterogeneity in study design and patient selection on the pooled effect estimate, we performed several sensitivity analyses. In general, exclusion of any single study from the analysis did not substantively alter the overall result of our analysis. Additionally, when data from the 11 studies $^{1-5, E 3, E 4, E 7, E 8, E 11, E 12}$ with similar preoperative variables in both groups were pooled $(\mathrm{N}=393)$, EVR was associated with a $62 \%$ reduction in mortality relative to OR that remained statistically significant $(7.5 \%$ vs $24.1 \%$; odds ratio, $0.38 ; 95 \%$ CI, $0.20-0.73 ; P<.01)$ (Figure 1 , lower panel). There was neither study heterogeneity of results $(P=.91)$ nor evidence of significant publication bias $(P=.24)$. 